\title{
Orientation dependence of NO sticking and scattering at $\mathrm{Pt}(100)$
}

\author{
H. Müller, G. Zagatta, N. Böwering, U. Heinzmann \\ Fakultät für Physik, Universität Bielefeld, D-33615 Bielefeld, Germany
}

Received 3 February 1994; in final form 29 March 1994

\begin{abstract}
Gas-phase oriented NO molecules are focused onto a $\mathrm{Pt}(100)$ surface in a supersonic molecular beam. The total number of the scattered and desorbed NO molecules is determined from recordings of the relative partial NO pressure. This signal shows a strong dependence on the initial NO orientation (preferential Nend or O-end collisions) and surface temperature. Apart from a direct chemisorption channel, the results show that there may be an orientation-dependent effect for the NO molecules trapping into a precursor state. Both channels occur in favor of $\mathrm{N}$-end collisions at surface temperatures between $T_{\mathrm{s}}=-120^{\circ} \mathrm{C}$ and $T_{\mathrm{s}}=100^{\circ} \mathrm{C}$.
\end{abstract}

\section{Introduction}

Depending on the surface temperature, the dynamics of the adsorption process run through several channels. At low surface temperatures the probability of direct chemisorption is high, whereas at high temperatures scattering dominates. In between those two possibilities, trapping into a physisorbed state becomes efficient.

In order to study this transition region of the adsorption channels, the temperature dependence of the $\mathrm{NO} / \mathrm{Pt}(100)$ adsorption has been investigated using a quadrupole mass spectrometer, which records the scattered and desorbed NO molecules from the $\mathrm{Pt}(100)$ surface. To resolve the influence of the orientation of the incoming NO molecules, the hexapole/orientation field technique was used. That is to say, the NO molecules are gas-phase oriented with respect to the surface normal approaching either preferentially with the $\mathrm{N}$-end or the $\mathrm{O}$-end. This method was already used in the extreme surface temperature regions for other adsorption systems: At low surface temperatures the NO sticking, NO induced $\mathrm{CO}$ de- sorption and NO scattering behaviour was examined for the systems $\mathrm{NO} / \mathrm{Ni}(100)$ and $\mathrm{NO} / \mathrm{CO} / \mathrm{Ni}(100)$ [1-3]. The results have shown that the initial molecular orientation has a pronounced influence on the adsorption process. In the case of $\mathrm{NO} / \mathrm{Ni}(100)$ the sticking probability for $\mathrm{N}$-end collisions has been found to be higher than for O-end collisions. After the collision process the $\mathrm{NO}$ is bound with the $\mathrm{N}$-end pointing towards the surface [4-6]. On the other side, the Kleyn and Bernstein groups have carried out scattering experiments at high surface temperatures $\left(>250^{\circ} \mathrm{C}\right)$ for the systems $\mathrm{NO} / \mathrm{Ag}(111)$, NO/ $\mathrm{Pt}(111)$ and $\mathrm{CH}_{3} \mathrm{~F} / \mathrm{graphite}$ [7-10] and found that scattering parameters, such as final rotational states of NO after the collision, depend strongly on the initial NO orientation. It is the purpose of this Letter to present sticking and scattering results of oriented NO at $\mathrm{Pt}(100)$, which is an intermediate system in between $\mathrm{Ni}(100)$ and $\mathrm{Pt}(111)$ referring to used surface temperatures. The dynamics of the adsorption process have been investigated by various methods. For the NO/Pt (100) system low energy electron diffraction (LEED) [11], infrared reflection absorp- 
tion spectroscopy (IRAS) [12], thermal desorption spectroscopy (TDS) [13], electron energy loss spectroscopy (EELS) [14], X-ray photoemission spectroscopies (XPS and UPS) [15], and theoretical investigations [16] have already been carried out. In comparison to the nickel results mentioned above, the platinum substrate provides two main differences. Firstly, the initial sticking probabilities $S_{0}$ of NO on $\mathrm{Ni}(100)$ were determined to lie between 0.4 and 0.7 for surface temperatures below $100^{\circ} \mathrm{C}[17,18]$; in the case of $\mathrm{NO} / \mathrm{Pt}(111) S_{0}$ was experimentally found to be larger than $0.7[19,20]$ and theoretically confirmed $[21,22]$ at incident translational energies of NO below $0.5 \mathrm{eV}$. Unfortunately, the sticking probability of NO on $\mathrm{Pt}(100)$ has still not been investigated. Secondly, the mobility of $\mathrm{NO}$ on $\mathrm{Pt}(100)$ is much higher than on $\mathrm{Ni}(100)$ (see the beginning of section 3 ).

\section{Experimental}

The experimental setup has been described in detail in Refs. [1-3]. Taking advantage of the hexapole technique [23], NO molecules are state selected, focused, and subsequently oriented in a homogeneous electric field [24] before colliding with the surface. Depending on the polarity of the electric field $E$ in front of the surface, two different orientation configurations can be achieved: preferential $\mathrm{N}$-end and preferential O-end collisions. In contrast to previous investigations employing a continuous beam source $[1,3]$, the source was now replaced by a pulsed nozzle in order to decrease the rotational temperature of the NO molecules. Since the degree of orientation depends strongly on the rotational state distribution of the NO molecules [10], an increase of the previous degree of orientation of $\overline{\langle\cos \Phi\rangle}=12 \%$ [1] can be expected. For instance, theoretically, NO molecules in the ${ }^{2} \Pi_{1 / 2}\left|J=\frac{1}{2}, \Omega=\frac{1}{2}, M_{J}=\frac{1}{2}\right\rangle$ ground state can reach $\overline{\langle\cos \Phi\rangle}=33.3 \%$ and the $\mid J=\frac{3}{2}, \Omega=\frac{1}{2}, M_{J}=$ $\left.\frac{3}{2}\right\rangle$ state can result in $\overline{\langle\cos \Phi\rangle}=20 \%$ in the limit of high electric field strength. The nozzle is operated with a gas mixture of $20 \% \mathrm{NO}, 35 \% \mathrm{He}$ and $45 \% \mathrm{Ne}$ and at a temperature of $T_{\text {nozzle }}=90^{\circ} \mathrm{C}$ resulting in a translational energy of $E_{\text {trans }}=150 \mathrm{meV}$. The speed ratio was determined to be $S=20$. The integral number of all NO molecules leaving the surface, is de- tected from the NO partial pressure $p_{\mathrm{s}, \mathrm{d}}$ (the subscript indicates scattering-desorption) with a quadrupole mass analyzer (QMA) located behind the target and thus shielded from the direct beam. Therefore, the signal $p_{\mathrm{s}, \mathrm{d}}$ contains the scattered as well as the desorbed molecules.

In order to achieve a clean, reproducibly prepared platinum surface, the preparation process is fully automated by programmable power supplies for the electron impact heating and by stepper motors mounted on the gas inlet valves [25]. The cleaning procedures included are $\mathrm{Ar}^{+} / \mathrm{Ne}^{+}$sputtering (once a day), heating in $\mathrm{O}_{2}\left(5 \times 10^{-8} \mathrm{mbar}, 5 \mathrm{~min}, 350^{\circ} \mathrm{C}\right)$ and flashing $\left(700^{\circ} \mathrm{C}\right)$. Low energy electron diffraction (LEED) shows the well-known hex-superstructure pattern $[26,27]$. The reproducibility of the preparation procedure is important because measurements for the three configurations ( $\mathrm{N}$-end collisions, unoriented, O-end collisions) with the same initial conditions have to be carried out in sequence. In addition, the substrate temperature was computer controlled and kept constant during the adsorption process.

\section{Results and discussion}

To compare the behaviour of NO mobility of $\mathrm{Pt}(100)$ with $\mathrm{Ni}(100)$, which has been investigated in previous experiments [1-3], we carried out investigations using a modified King and Wells technique [28] at first. The diffusion parameters for NO were estimated by exposing the surfaces to NO for a certain time, pausing and then exposing again, while recording the NO molecules leaving the surface. In the limiting case of high diffusion rates, the results of the measurements were found to be the same after each exposure. This has been observed for $\operatorname{Pt}(100)$. In the other limiting case of low diffusion, the coverage continues to increase. This has been found in the case of $\mathrm{Ni}(100)$.

NO already desorbs from the Pt(100) surface at $-120^{\circ} \mathrm{C}<T_{\mathrm{s}}<100^{\circ} \mathrm{C}$ as shown in TDS spectra $[13,25]$. Therefore, the surface diffusion parameter also depends on the surface temperature. Since NO is exposed to the surface in a focused supersonic beam, the effective adsorption spot is small $(\varnothing<1$ $\mathrm{mm})$ in relation to the crystal size $(\varnothing=12 \mathrm{~mm})$. Up 
to saturation coverage, the NO movement on $\mathrm{Ni}(100)$ is negligible. In contrast, NO is mobile on $\mathrm{Pt}(100)$, which makes it difficult to evaluate the coverage dependence of the sticking probability. In this Letter, we report on measurements of $p_{s, \mathrm{~d}}$ as a function of the surface temperature $T_{\mathrm{s}}$ and of the orientation of the incoming molecules. As already described in section 2 , the signal $p_{\mathrm{s}, \mathrm{d}}$ contains the scattered as well as the desorbed molecules and is normalized to the intensity of the direct beam. $p_{\mathrm{s}, \mathrm{d}}^{\mathrm{U}}$, $p_{\mathrm{s}, \mathrm{d}}^{\mathrm{N}}$ and $p_{\mathrm{s,d}}^{\mathrm{O}}$ stand for the partial pressures of NO for unoriented, preferential $\mathrm{N}$-end and preferential $\mathrm{O}$-end collisions, respectively.

In order to check the degree of orientation, $p_{\mathrm{s}, \mathrm{d}}$ is investigated as a function of the field strength at a substrate temperature of $T_{\mathrm{s}}=50^{\circ} \mathrm{C}$, as shown in the upper part of Fig. 1. While the constant (field strength independent) value for the unoriented molecules $\left(p_{\mathrm{s}, \mathrm{d}}^{\mathrm{U}}\right)$ lies in-between the two values for the oriented molecules ( $p_{\mathrm{s}, \mathrm{d}}^{\mathrm{N}}$ and $p_{\mathrm{s}, \mathrm{d}}^{\mathrm{O}}$ ) up to $E=10 \mathrm{kV} / \mathrm{cm}$, the mean of $p_{\mathrm{s}, \mathrm{d}}^{\mathrm{N}}$ and $p_{\mathrm{s}, \mathrm{d}}^{\mathrm{O}}$ deviates from $p_{\mathrm{s}, \mathrm{d}}^{\mathrm{U}}$ at higher field strength. When increasing the field to more than $E=10 \mathrm{kV} / \mathrm{cm}$, both values for oriented molecules increase. In the case examined here, the results from measurements on unoriented molecules have to be equal to the mean values from measurements with opposite orientations. It may be that the additional phenomenon at a high electric field can be explained by field-induced desorption $[29,30]$. This effect limits the field strength to a value of $E=12 \mathrm{kV} / \mathrm{cm}$, at which the second set of experiments has been carried out.

In the lower part of Fig. 1 the asymmetry is displayed, which is defined as $A_{\mathrm{s}, \mathrm{d}}=\left(p_{\mathrm{s}, \mathrm{d}}^{\mathrm{O}}-p_{\mathrm{s}, \mathrm{d}}^{\mathrm{N}}\right) /$ $\left(p_{\mathrm{s}, \mathrm{d}}^{\mathrm{O}}+p_{\mathrm{s}, \mathrm{d}}^{\mathrm{N}}\right)$. It corresponds to the values given in the upper part of Fig. 1. The asymmetry increases as a function of the field strength. Since this increase from $E=10 \mathrm{kV} / \mathrm{cm}$ up to $E=12 \mathrm{kV} / \mathrm{cm}$ is very small, the maximum degree of orientation is almost reached. This explanation is compatible with the corresponding results of Ref. [3], where the degree of orientation of 0.17 was determined at a field strength of $E=6.4 \mathrm{kV} / \mathrm{cm}$ with the same beam parameters. Fitting the results to the theoretical curve [1], a mean degree of orientation of about $\overline{\langle\cos \Phi\rangle}=30 \%$ is achieved.

$p_{s, \mathrm{~d}}$ versus temperature for both orientations is given in the upper part of Fig. 2. All temperatures
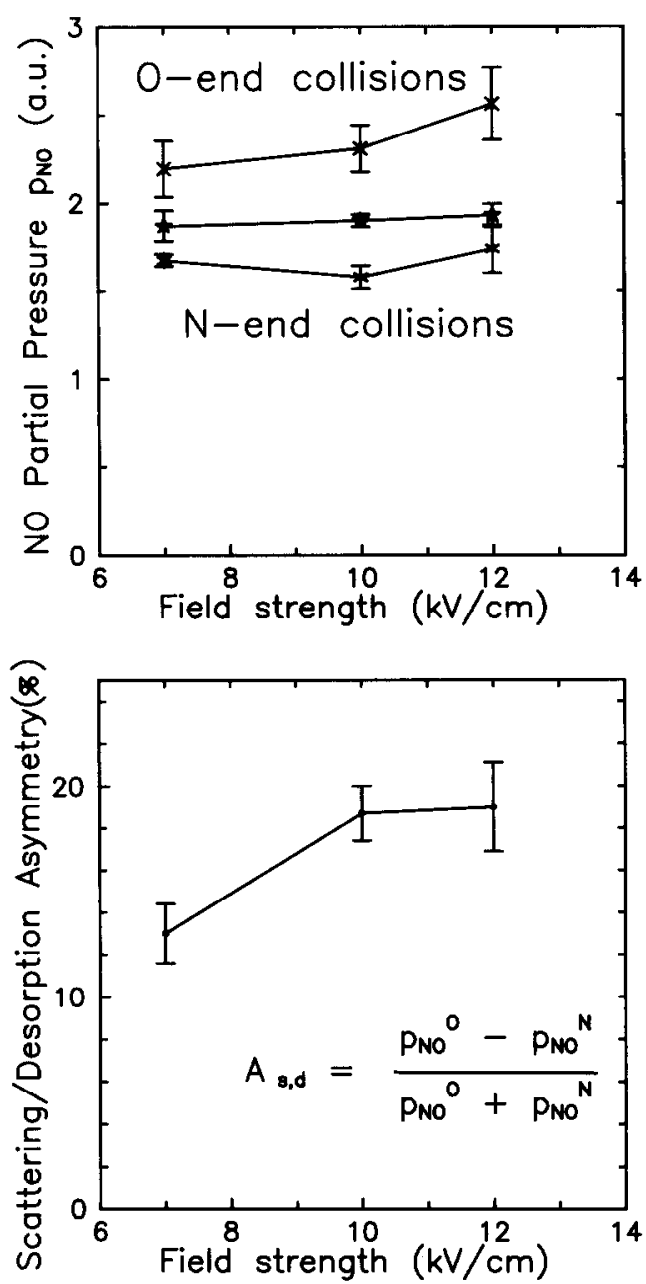

Fig. 1. Upper part: Partial pressure of $\mathrm{NO} p_{\mathrm{a}, \mathrm{d}}$ leaving (scattering and desorbing from) the clean $\mathrm{Pt}(100)$ surface, as a function of field strength and NO orientation (O-end collisions, random collisions and N-end collisions) at $T_{a}=50^{\circ} \mathrm{C}$. Lower part: Corresponding orientation asymmetry of the partial pressure of NO given by $A_{\mathrm{a}, \mathrm{d}}=\left(p_{\mathrm{a}, \mathrm{d}}^{\mathrm{Q}}-p_{\mathrm{a}, \mathrm{d}}^{\mathrm{N}}\right) /\left(p_{\mathrm{s}, \mathrm{d}}^{\mathrm{O}}+p_{\mathrm{s}, \mathrm{d}}^{\mathrm{N}}\right)$.

investigated are below the dissociation barrier of NO on $\operatorname{Pt}(100)$, which has been found to be $T_{\mathrm{s}} \approx 125^{\circ} \mathrm{C}$ $[13,15,31]$. The $p_{\mathrm{s}, \mathrm{d}}$ curves show a weak peak located slightly below $T_{\mathrm{s}}=50^{\circ} \mathrm{C}$. This supports the assumption that desorption plays an important role, although the data were always taken immediately after exposure to the NO beam. Comparing the lifetime of precursor states with the rise time in the experiments (1 s), an adsorption-desorption equilibrium can be presumed. Within this rise time of one second the NO coverage stays almost constant, particularly as the 

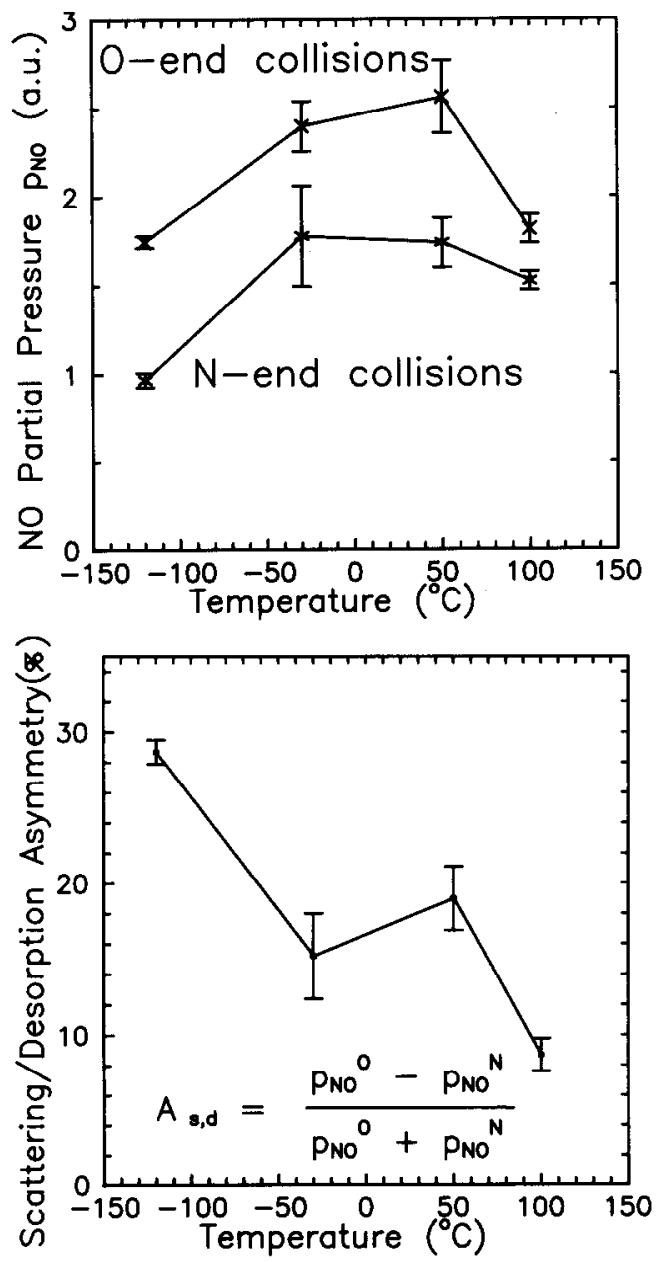

Fig. 2. Upper part: Partial pressure of NO $p_{a, d}$ leaving (scattering and desorbing from) the clean $\mathrm{Pt}(100)$ surface, as a function of temperature and NO orientation at a field strength of $E=12 \mathrm{kV} /$ $\mathrm{cm}$. Lower part: Orientation asymmetry of the partial pressure of NO given by $A_{\mathrm{a}, \mathrm{d}}=\left(p_{\mathrm{a}, \mathrm{d}}^{\mathrm{O}}-p_{\mathrm{a}, \mathrm{d}}^{\mathrm{N}}\right) /\left(p_{\mathrm{g}, \mathrm{d}}^{\mathrm{O}}+p_{\mathrm{a}, \mathrm{d}}^{\mathrm{N}}\right)$ corresponding to the upper part.

diffusion rate of NO on $\mathrm{Pt}(100)$ is high. O-end collisions always exhibit a higher yield of molecules leaving the surface.

The desorption peak is accompanied by a background which can be assigned to the scattered molecules. This background slightly increases from $T_{3}=-120^{\circ} \mathrm{C}$ to $T_{s}=100^{\circ} \mathrm{C}$, which is in accordance with the decreasing initial sticking probability as a function of the temperature [31]. Therefore, we assume a superposition of scattering and desorption. Another explanation for the decrease of the NO yield at $T_{\mathrm{s}}=100^{\circ} \mathrm{C}$ might be that NO starts to dissociate, although, as already mentioned, the dissociation barrier of $\mathrm{NO}$ on $\mathrm{Pt}(100)$ should be above $T_{\mathrm{s}}=125^{\circ} \mathrm{C}$. Consequently, the dissociated NO would not be detected at mass 30 . This second explanation could not be excluded, however, since the NO data were taken at the very beginning of the run ( $1 \mathrm{~s}$ ) and an analysis of the gases on the surface at such low coverages was not feasible.

The asymmetry corresponding to the upper part of Fig. 2 is given in the lower part of Fig. 2. Taking the degree of orientation $\overline{\langle\cos \Phi\rangle}=30 \%$ into account, for the results at $T_{\mathrm{s}}=-120^{\circ} \mathrm{C}$ the measured asymmetry of almost $30 \%$ would lead to a normalized asymmetry of almost $100 \%$ for perpendicular collisions. In this gedanken experiment, in which all molecules rigidly oriented collide either with the N-end or the $\mathrm{O}$ end towards the surface, the asymmetry leads to the assumption that the scattering and desorption signal for $\mathrm{N}$-end collisions is nearly zero. As a consequence of the definition of an asymmetry, the value can only be $100 \%$, if one of the signals is zero. If $\mathrm{N}$-end collisions do not contribute to either scattering or desorption, these perfectly $\mathrm{N}$-end oriented NO molecules will stick, since scattering/desorption and sticking are complementary. However, this does not mean that $\mathrm{O}$ end oriented molecules in the scattering/desorption signal do not stick or, in other words, that scattering/ desorption asymmetry and sticking asymmetry have the same value $(100 \%)$. The direct chemisorption channel is most likely responsible for the very high sticking probability of the $\mathrm{N}$-end oriented molecules. The asymmetry decreases with increasing surface temperature, because the number of molecules that do not stick increases for both orientations investigated. However, the value at $T_{\mathrm{s}}=50^{\circ} \mathrm{C}$, at which the desorption occurs, does not fit in this simple picture. Increasing the surface temperature, the trapping probability into precursor states becomes more efficient. Theoretical investigations including precursor states have been carried out for the Kisliuk model [32-34] and desorption kinetics with intermediate states [35-37]. Since the asymmetry value at $T_{\mathrm{s}}=50^{\circ} \mathrm{C}$ is slightly higher, this trapping can be assumed to be orientation dependent. Both channels, direct chemisorption and trapping into the precursor state, occur in favour of $\mathrm{N}$-end collisions. This cor- 
responds to the orientation of NO, which is bound with the $\mathrm{N}$-end pointing to the $\mathrm{Pt}(100)$ surface [14].

\section{Conclusion}

In conclusion, the adsorption of $\mathrm{NO}$ on $\mathrm{Pt}(100)$ strongly depends on the initial gas-phase orientation of the incoming NO molecules. Both trapping into a precursor state and direct chemisorption occur in favour of $\mathrm{N}$-end collisions between $T_{\mathrm{s}}=-120^{\circ} \mathrm{C}$ and $T_{\mathrm{s}}=100^{\circ} \mathrm{C}$.

\section{Acknowledgement}

Financial support by DFG(SFB 216) and the Commission of the European Communities is gratefully acknowledged.

\section{References}

[1] G.H. Fecher, N. Böwering, M. Volkmer, B. Pawlitzky and U. Heinzmann, Surface Sci. Letters 230 (1990) L169.

[2] G.H. Fecher, M. Volkmer, B. Pawlitzky, N. Böwering and U. Heinzmann, Vacuum 41 (1990) 265.

[3] H. Müller, B. Dierks, F. Hamza, G. Zagatta, G.H. Fecher, N. Böwering and U. Heinzmann, Surface Sci. 269/270 (1992) 207.

[4] J. Stöhr and R. Jaeger, Phys. Rev. B 26 (1982) 4111.

[5] A. Sandell, A. Nilsson and N. Mårtensson, Surface Sci. Letters 241 (1991) L1.

[6] A. Sandell, A. Nilsson and N. Mårtensson, Surface Sci. 251/ 252 (1991) 971.

[7] E.W. Kuipers, M.G. Tenner, A.W. Kleyn and S. Stolte, Nature 334 (1988) 420.

[8] T.J. Curtiss and R.B. Bernstein, Chem. Phys. Letters 161 (1989) 212.

[9] E.W. Kuipers, M.G. Tenner, A.W. Kleyn and S. Stolte, Phys. Rev. Letters 62 (1989) 2152.
[10] M.G. Tenner, E.W. Kuipers, W.Y. Langhout, A.W. Kleyn, G. Nicolasen and S. Stolte, Surface Sci. 236 (1990) 151.

[11] K. Mase and Y. Murata, Surface Sci. 277 (1992) 97.

[12] P. Gardner, M. Tüshaus, R. Martin and A.M. Bradshaw, Surface Sci. 240 (1990) 112.

[13] R.J. Gorte and L.D. Schmidt, Surface Sci. 109 (1981) 367.

[14] G. Pirug, H.P. Bonzel, H. Hopster and H. Ibach, J. Chem. Phys. 71 (1979) 593.

[15] H.P. Bonzel and G. Pirug, Surface Sci. 62 (1977) 45.

[16] W.F. Banholzer, Y.O. Park, K.M. Mak and R.I. Masel, Surface Sci. 128 (1983) 176.

[17] G.H. Fecher, Ph.D. Thesis, Universität Bielefeld (1990).

[18] A.V. Hamza, P.M. Ferm, F. Budde and G. Ertl, Surface Sci. 199 (1988) 13.

[19] C.T. Campbell, G. Ertl and J. Segner, Surface Sci. 115 (1982) 309.

[20] J.K. Brown and A.C. Luntz, Chem. Phys. Letters 204 (1993) 451.

[21] C.W. Mulhausen, L.R. Williams and J.C. Tully, J. Chem. Phys. 83 (1985) 2594.

[22] C. Haug, W. Brenig and T. Brunner, Surface Sci. 265 (1992) 56.

[23] K.H. Kramer and R.B. Bernstein, J. Chem. Phys. 42 (1965) 767.

[24] D. van den Ende and S. Stolte, Chem. Phys. 89 (1984) 121.

[25] G. Zagatta, Diploma Thesis, Universität Bielefeld (1992).

[26] P. Heilmann, K. Heinz and K. Müller, Surface Sci. 83 (1979) 487.

[27] E. Lang, PhD Thesis, Friedrich-Alexander-Universität Erlangen-Nürnberg (1982).

[28] D.A. King and M.G. Wells, Surface Sci. 29 (1972) 454.

[29] H.J. Kreuzer and L.C. Wang, J. Chem. Phys. 93 (1990) 6065.

[30] J.H. Block, in: Chemistry and physics of solid surfaces, Vol. 4, eds. R. Vanselow and R. Howe (Springer, Berlin, 1982) pp. $407 f f$.

[31] H.P. Bonzel, G. Brodén and G. Pirug, J. Catal. 53 (1978) 96.

[32] P. Kisliuk, J. Phys. Chem. Solids 3 (1957) 95.

[33] P. Kisliuk, J. Phys. Chem. Solids 5 (1958) 78.

[34] K. Schönhammer, Surface Sci. Letters 83 (1979) L633.

[35] A. Cassuto and D.A. King, Surface Sci. 102 (1981) 388.

[36] D.A. King, Surface Sci. 64 (1977) 43.

[37] G. Gorte and L.D. Schmidt, Surface Sci. 76 (1978) 559. 